\title{
INNER FACTORS AND BLASCHKE PRODUCTS ${ }^{1}$
}

\section{NORMAN RUSHFIELD}

1. Introduction. Let $L_{2, v}$ denote the space of functions on the positive real axis into a separable Hilbert space $V$, such that $\int_{0}^{\infty}|f(x)|^{2} d x$ $<\infty$ and for every $u \in V,(f(x), u)$ is measurable. Let $L_{2,0}$ denote the space of Fourier transforms

$$
\hat{f}(t)=\int_{0}^{\infty} f(x) e^{i t x} d x
$$

where $f \in L_{2, v}$ and $d x$ denotes $d x / 2 \pi$. The functions in $\hat{L_{2, v}}$ can be extended to analytic functions in the upper half plane. We denote this space of analytic functions by $H_{v}$. By the Paley-Wiener theorem, $H_{v}$ is characterized by the property that $h \in H_{v}$ if and only if for some constant $M$ and every $y>0, \int_{-\infty}^{\infty}(h(x+i y), h(x+i y)) d x<M . H_{v}$ is a Hilbert space with inner product $(f, g)_{1}=\int_{0}^{\infty}(f(x), g(x)) d x$.

Let $T_{s}$ (for fixed $s>0$ ) be the left translation operator on $L_{2, v}$, $T_{s} f(x)=f(x+s)$. The family $\left\{T_{s} \mid s>0\right\}$ is a semigroup of operators.

Let $\tau_{s}$ (for fixed $s>0$ ) be the right translation operator on $L_{2, v}$ :

$$
\tau_{s} g(x)= \begin{cases}g(x-s), & \text { for } x-s \geqq 0, \\ 0, & \text { for } x-s<0 .\end{cases}
$$

The family $\left\{\tau_{s} \mid s>0\right\}$ is a semigroup of isometric operators.

A subspace $l$ of $L_{2, v}$ is said to be left invariant (an $l$-space) if for every $f \in l,\left\{T_{s} f \mid s>0\right\} \subseteq l$.

A subspace $r$ of $L_{2, v}$ is said to be right invariant (an $r$-space) if for every $g \in r,\left\{\tau_{s} g \mid s>0\right\} \subseteq r$. It is easily seen that the orthogonal complement of an $r$-space is an $l$-space and vice-versa.

An inner factor is an operator valued function defined and analytic in the upper half plane such that for each $z, F(z): W \rightarrow V$ (where $W$ and $V$ are separable Hilbert spaces), $\|F(z)\| \leqq 1$, and for almost all real $z, F(z)$ is an isometry. If $V$ is finite dimensional, and $W=V$ then $\operatorname{det} F$ (defined as the determinant of the matrix $\left(F(z) u_{i}, u_{i}\right)$, where $u_{i}$ is an orthonormal basis for $V$ ) is a scalar inner factor.

Let $R$ denote the Fourier transform space of an $r$-space. An $R$ space is characterized as being invariant under multiplication by

Received by the editors February 16, 1965.

1 This paper is a portion of the author's doctoral dissertation. The author would like to express his thanks to Professor P. D. Lax for suggesting the problems considered and for his guidance in the course of this work. 
$e^{i s z}$ for every $s>0$. The following result has been proved by Lax [4], [5] and by Halmos [2]:

THEOREM A. Every (nonzero) closed $R$-space can be represented in the form $R_{v}=F^{v} H_{w}$, where $F^{v}$ is an inner factor and $F^{v}(z): W \rightarrow V$.

The inner factor corresponding to an $R$-space is unique to within multiplication on the right by a constant unitary operator. By regarding two inner factors as equivalent if they differ on the right by a constant unitary operator, we obtain a one-to-one correspondence between nonzero closed $R$-spaces and inner factors. This correspondence carries over to closed $l$-spaces $\left(l \subset L_{2, v}\right)$, where $R$ is the Fourier transform space of $r\left(=l^{\perp}\right)$ and will be denoted by a common subscript $l_{a} \sim F_{a}$ (or lack of subscript, $l \sim F$ ). From the division theory of $[4]$, we have

THEOREM B. $l_{a} \subseteq l_{b}$ if and only if there exists an inner factor $F_{c}$ such that $F_{b}=F_{a} F_{c}$.

A (generalized) exponential is a function $f \in L_{2, v}$ of the form $f=p(x) e^{i \lambda x}$ where $p(x)$ is a polynomial with coefficients in $V$, and $\operatorname{Im} \lambda>0$. We define the order of the exponential $f$ as the degree of $p(x) ; \lambda$ is called the exponent belonging to the exponential $f$.

Definition. An inner factor $F$ is said to be a Blaschke product if $l(\sim F)$ is spanned by the exponentials contained in $l$.

We show that in the case of scalar inner factors, our definition of a Blaschke product is equivalent to the standard definition.

Let $\left[v_{j} \mid j \in J\right]$ denote the smallest closed $l$-space containing $\left\{v_{j} \mid j \in J\right\}$. Let $l=\left[x^{k_{j}} e^{i \lambda_{j} x} \mid j \in J\right]$; we note that

$$
\left[x^{k_{j}} e^{i \lambda_{j} x} \mid j \in J\right]=\left[p_{k_{j}}(x) e^{i \lambda_{j} x} \mid j \in J\right],
$$

where $p_{k_{j}}$ is a polynomial of degree $k_{j}$.

Let $F$ be a Blaschke product according to the standard definition. We will show that $l(\sim F)$ has a basis of exponentials. Let $g$ be an arbitrary element of $l^{\perp}$, and $F h=\hat{g}$. If $F$ has a zero of order $m_{j}$ at $z=-\bar{\lambda}_{j}$, then it follows from the equation

$$
(F h)^{k_{j}}\left(-\bar{\lambda}_{j}\right)=\int_{0}^{\infty} g(x)(i x)^{k_{j}} e^{-i \bar{\lambda}_{j} x} d x,
$$

that $l_{e}\left(=\left[x^{m_{j}-1} e^{i \lambda_{j} x} \mid j \in J\right]\right) \subseteq l$.

We need only show that $l \subseteq l_{e}$. If we substitute $F_{e}\left(\sim l_{e}\right)$ for $F$ in equation (1.1), and let $g$ be an arbitrary element of $l_{e}^{\perp}$, we see that $F$ has a zero of order $m_{j}$ at $z=-\bar{\lambda}_{j}$. It then follows that $F_{e} / F$ is an 
inner factor. Thus $F_{e}$ is of the form $F_{e}=F F_{c}$, so that by Theorem B, $l \subseteq l_{e}$.

Conversely, we let $l=\left[x^{m_{j}-1} e^{i \lambda_{j} x} \mid j \in J\right]$, and show that $F(\sim l)$ is a standard Blaschke product. It follows from Equation (1.1) that $F$ has a zero of order $m_{j}$ at $z=-\bar{\lambda}_{j}$. Let $F_{e}$ be the standard Blaschke product with zeros of order $m_{j}$ at $Z=-\bar{\lambda}_{j}$. Then $F / F_{e}$ is an inner factor, so that $F=F_{e} F_{a}$. According to Theorem B, $l_{e} \subseteq l$. From equation (1.1) we see that $l \subseteq l_{e}$, so that $l_{e}=l$. This implies that $F_{a}$ is constant, and completes the proof.

Definition. An inner factor $F(z)$ is said to be nonsingular if $\operatorname{det} F(z)$ has no zeros (in the upper half plane).

The following results are well known, but we sketch the proof of Theorem $\mathrm{D}$ for the sake of completion:

THEOREM C. Every scalar inner factor $F$ can be factored in the form $F=F_{e} F_{n}$, where $F_{e}$ is a Blaschke product and $F_{n}$ is nonsingular. $F_{e}$ and $F_{n}$ are uniquely determined to within a constant unitary factor.

Theorem D. If $F$ is a scalar Blaschke product, and $F=F_{a} F_{b}$ then $F_{a}$ and $F_{b}$ are Blaschke products.

Proof. We factor $F_{a}$ and $F_{b}$ as in Theorem $\mathrm{C}$ to obtain $F_{a}=F_{1} F_{2}$, $F_{b}=F_{3} F_{4}$ (where $F_{1}$ and $F_{3}$ are Blaschke products and $F_{2}$ and $F_{4}$ are nonsingular). We must show that $F_{2}$ and $F_{4}$ are constant. We have $F=\left(F_{1} F_{3}\right)\left(F_{2} F_{4}\right)$. From Theorem $\mathrm{C}$ we see that $F_{2} F_{4}$ is a constant unit. It then follows from the properties of inner factors that $F_{2}$ and $F_{4}$ are constant units.

2. Results. We consider the case where $V=W$ and is finite dimensional, and we prove the following two theorems:

THEOREM 1. Every inner factor $F$ can be factored in the form $F=F_{e} F_{n}$ where $F_{e}$ is a Blaschke product and $F_{n}$ is nonsingular.

Theorem 2. Fis a Blaschke product if and only if $\operatorname{det} F$ is a Blaschke product.

We now have a generalization of Theorem $\mathrm{D}$ in the

Corollary. If $F$ is a Blaschke product and $F=F_{a} F_{b}$ where $F_{a}$ and $F_{b}$ are inner factors, then $F_{a}$ and $F_{b}$ are Blaschke products.

To prove these results we need the following three lemmas; their proofs will be given in $\$ 3$.

Lemma 1. If $\operatorname{det} F$ is constant, then $F$ is a constant unitary operator. 
Lemma 2. Let $l_{\lambda}$ be the span of the exponentials (with exponent $\lambda$ ) which are contained in $l$, and let $F \sim l$. Then $\operatorname{dim} l_{\lambda}$ equals the order of the zero of $\operatorname{det} F(z)$ at $z=-\bar{\lambda}$.

Lemma 3. Let $\beta(z)$ be the Blaschke product factor of $\operatorname{det} F$. Let $l \sim F, l_{\beta} \sim \beta(z) I$, and let $l_{\theta}$ be the span of the exponentials which are contained in $l$. Then $l_{e} \subseteq l_{\beta}$.

Proof of Theorem 1. Let $l_{\boldsymbol{e}}$ be the span of the exponentials contained in $l(\sim F)$, and let $F_{e} \sim l_{e}$. According to Theorem B, there exists an inner factor $F_{n}$ such that $F=F_{e} F_{n}$. Taking determinants of both sides, we have $\operatorname{det} F=\operatorname{det} F_{e} \operatorname{det} F_{n}$. Let $d, d_{e}$, and $d_{n}$ denote the order of the zero at $z=-\bar{\lambda}$ of $\operatorname{det} F, \operatorname{det} F_{e}$, and $\operatorname{det} F_{n}$ respectively, so that $d=d_{e}+d_{n}$. We must show that $d_{n}=0$. Since $l$ and $l_{e}$ have the same number of linearly independent exponentials with exponent $\lambda$, it follows from Lemma 2 that $d=d_{e}$.

Proof of Theorem 2. Assume that det $F$ is a Blaschke product. We factor $F$ as in Theorem 1:F=F $F_{e} F_{n}$. We must show that $F_{n}$ is constant. Taking determinants, we have $\operatorname{det} F=\operatorname{det} F_{e} \operatorname{det} F_{n}$. By virtue of Theorem $\mathrm{D}$, $\operatorname{det} F_{n}$ is a Blaschke product. But since according to Theorem 1 , det $F_{n}$ has no zeros, it follows that $\operatorname{det} F_{n}$ is constant. Then by virtue of Lemma $1, F_{n}$ is constant.

Conversely, assume that $F$ is a Blaschke product. Let $\beta(z)$ be the Blaschke product factor of det $F$. Let $l \sim F$ and $l_{\beta} \sim \beta(z) I$. According to Lemma $3, l \subseteq l_{\beta}$. Then by Theorem B, there exists an inner factor $F_{a}$ such that $\beta(z) I=F F_{a}$. Taking determinants, we have $\beta^{n}(z)$ $=\operatorname{det} F \operatorname{det} F_{a}($ where $n=\operatorname{dim} V)$. By virtue of Theorem $\mathrm{D}$, $\operatorname{det} F$ is a Blaschke product.

\section{Proofs of lemmas.}

Proof of Lemma 1. Since det $F(z)(=c)$ is an inner factor, we have $\operatorname{det}\left(F^{*}(z) F(z)\right)=\bar{c} c=1$. Let $\left\{a_{j}(z) \mid j=1, \cdots, n\right\}$ be the eigenvalues of $F^{*}(z) F(z)$. Since $\left\|F^{*}(z) F(z)\right\| \leqq 1$ (for $\operatorname{Im} z>0$ ), and $F^{*}(z) F(z)$ is nonnegative, we have $0 \leqq a_{j}(z) \leqq 1(j=1, \cdots, n)$. It then follows from the equation $\operatorname{det}\left(F^{*}(z) F(z)\right)=\prod_{j=1}^{n} a_{j}(z)=1$ that all the eigenvalues of $F^{*}(z) F(z)$ are equal to one. Since $F^{*}(z) F(z)$ is symmetric, we thus have $F^{*}(z) F(z)=I$. Similarly $F(z) F^{*}(z)=I$, so that $F^{*}(z)$ $=F^{-1}(z)$. Since $F(z)$ is analytic and $F^{-1}(z)$ is continuous, $F^{-1}(z)$ is analytic. But if $F(z)$ and $F^{*}(z)\left(=F^{-1}(z)\right)$ are both analytic, it follows that $F(z)$ is constant.

The proof of Lemma 2 is based on four sublemmas:

Leмма 3.1. Let $l=\left[u e^{i \lambda x}\right]$ where $u$ is a given vector in $V$ and $\operatorname{Im}>0$. Then $F(\sim l)$ is (up to a constant unitary operator on the right) of the form 


$$
\begin{aligned}
F & =P+b(z) Q, \\
b(z) & =\frac{z+\bar{\lambda}}{z+\lambda},
\end{aligned}
$$

$Q$ is the orthogonal projection onto the one dimensional space spanned by $u$, and $P=I-Q$.

Proof. Let $l_{p} \sim P+b(z) Q$. Then

$$
\left((P+b(z) Q) h_{v}, u\right)=\left(h_{v}, P^{*} u\right)+b(z)\left(h_{v}, Q^{*} u\right)=b(z)\left(h_{v}, u\right) .
$$

Since $b(-\bar{\lambda})=0$, it follows from the equation

$$
0=\left((P+b(-\bar{\lambda}) Q) h_{v}(-\bar{\lambda}), u\right)=\int_{0}^{\infty}\left(a_{v}, u\right) e^{-\bar{i} x} d x
$$

(where $a_{v} \in l_{p}^{\perp}$ ) that $l\left(=\left[u e^{i \lambda x}\right]\right) \in l_{p}$. From Theorem B we obtain $P+b(z) Q=F F_{a}$ (where $\left.F \sim l\right)$. Taking determinants of both sides of the factorization equation we have $b(z)=\operatorname{det} F \cdot \operatorname{det} F_{a}$. It follows from Theorem $\mathrm{D}$ that either $\operatorname{det} F$ or $\operatorname{det} F_{a}$ is constant. If det $F$ were constant, then by Lemma $1, F$ would be a constant unitary operator. Clearly, this would imply that $l=\{0\}$. Thus by contradiction we see that $\operatorname{det} F_{a}$, and therefore $F_{a}$, must be constant, so that $l=l_{p}$.

Definition. An inner factor of the form (3.1) is called a prime inner factor (at $\lambda$ ).

Lemma 3.2. Let $F$ be an inner factor, and let $\operatorname{det} F$ have a zero of order at least $m$ at $z=-\bar{\lambda}$. Then $F$ can be factored in the form $F$ $=\left(\prod_{j=1}^{m} F_{j}\right) F_{a}$ for some inner factor $F_{a}$, where $\left\{F_{j} \mid j=1, \cdots, m\right\}$ are prime inner factors (at $\lambda$ ).

Proof. We use induction. Take $m=1$. Since $\operatorname{det} F(-\bar{\lambda})=0$, there exists a vector $u(u \neq 0)$ such that $F^{*}(-\bar{\lambda}) u=0$. Let $l \sim F$. It follows from the equation

$$
0=\left(h_{v}(-\bar{\lambda}), F^{*}(-\bar{\lambda}) u\right)=\left(F(-\bar{\lambda}) h_{v}(-\bar{\lambda}), u\right)=\int_{0}^{\infty}\left(a_{v}, u\right) e^{-i \bar{\lambda} x} d x
$$

(where $a_{v} \in l^{\perp}$ ) that $u e^{i \lambda x} \in l$. Let $l_{1}=\left[u e^{i \lambda x}\right]$, so that $l_{1} \subseteq l$. Then by Theorem B, we have $F=F_{1} F_{a}$, where $F_{1} \sim l_{1}$. By virtue of Lemma 3.1, $F_{1}$ is a prime inner factor (at $\lambda$ ).

We now assume that the lemma is true for $m=k$, and consider the case $m=k+1$. By our assumption we have $F=\left(\prod_{j=1}^{k} F_{j}\right) F_{a}$. Taking determinants, we have $\operatorname{det} F=b^{k}(z) \cdot \operatorname{det} F_{a}$. Since the order of the zero of $b^{k}(z)$ at $z=-\bar{\lambda}$ is $k$, we must have $\operatorname{det} F_{a}(-\bar{\lambda})=0$. Then, 
as shown above, $F_{a}=F_{k+1} F_{b}$ for some inner factor $F_{b}$, where $F_{k+1}$ is a prime inner factor (at $\lambda$ ).

Lemma 3.3. The space $l=\left[u x^{m-1} e^{i \lambda x} \mid u \in V\right]$ corresponds to the inner factor $F_{b}=b^{m}(z) I$.

Let $l_{1}=\left[u x^{m-1} e^{i \lambda x} \mid u \in V\right]$. It follows from the equation

$$
\left(F(z) h_{v}, u\right)^{(k)}=\int_{0}^{\infty}\left(a_{v}, u\right)(i x)^{k} e^{i z x} d x,
$$

for $a_{v} \in l_{1}^{\perp}$, and $F=F_{1}\left(\sim l_{1}\right)$, that $F_{1}$ has a zero of order at least $m$ at $z=-\bar{\lambda}$. That is, $F_{1}^{(\mathbf{k})}(-\bar{\lambda})=0, k=0,1, \cdots, m-1$. Then we can factor $F_{1}$ in the form $F_{1}=b^{m}(z) I \cdot F_{c}$, where $F_{c}$ is an inner factor. We need only show that $F_{c}$ is constant. Let $l_{b} \sim F_{b}=b^{m}(z) I$. It is easy to see from equation (3.3), for $\gamma_{v}=l_{b}^{\perp}$, and $F=F_{b}$, that $l_{1} \subseteq l_{b}$. Then by Theorem $\mathrm{B}$ we have $b^{m}(z) I=\left(b^{m}(z) I \cdot F_{c}\right) F_{a}$. It follows that $F_{c}$ is a constant unitary operator.

LemMA 3.4. $l(\sim F)$ consists of exponentials with exponent $\lambda$ and is finite dimensional if and only if $\operatorname{det} F=c b^{m}(z) . A l s o$, if $\operatorname{det} F=c b^{m}(z)$, then $\operatorname{dim} l=m$.

Let $l$ consist of exponentials with exponent $\lambda$, and let $q-1$ be the order of the highest order exponential contained in $l$. Let $l_{b}$ $=\left[u x^{q-1} e^{i \lambda x} \mid u \in V\right]$. Then $l \subseteq l_{b}$. According to Lemma 3.3, $b^{q}(z) I \sim l_{b}$. By virtue of Theorem $\mathrm{B}$, we have $b^{q}(z) I=F F_{a}$ (where $\left.F \sim l\right)$. Taking determinants, we obtain $b^{a n}(z)=\operatorname{det} F \cdot \operatorname{det} F_{a}($ where $n=\operatorname{dim} V)$. It follows from Theorem $\mathrm{D}$ that det $F$ is some power of $c b(z)$.

Conversely, let $\operatorname{det} F=c b^{m}(z)$. According to Lemma 3.2 we can factor $F$ in the form

$$
F=\left(\prod_{j=1}^{m} F_{j}\right) F_{a}
$$

where $F_{j}$ is the prime inner factor $P_{j}+b(z) Q_{j}$. By taking determinants of both sides of equation (3.4), we see that det $F_{a}=c$. Then by virtue of Lemma $1, F_{a}$ is a constant unitary operator. Let $E_{j}=Q_{j}+b(z) P_{j}$ $(j=1, \cdots, m)$. Clearly each $E_{j}$ is an inner factor. Since

$$
\left(\prod_{j=1}^{m} F_{j}\right) F_{a} F_{a}^{*}\left(\prod_{j=0}^{m-1} E_{m-j}\right)=b^{m}(z) I,
$$

it follows from Theorem B that $l \subseteq l_{b}$. It then follows from Lemma 3.3 that $l$ consists of exponentials with exponent $\lambda$. 
Again, we assume that $\operatorname{det} F=c b^{m}(z)$. We will show that $\operatorname{dim} l=m$. As shown in the previous paragraph,

$$
l\left(\sim \prod_{j=1}^{m} F_{j}\right) \subseteq l_{b}\left(\sim b^{m}(z) I\right)
$$

According to Theorem B, we have $b^{m}(z) I=\left(\prod_{j=1}^{m} F_{j}\right) F_{a}$. Taking determinants, we have $b^{m n}(z)=b^{m}(z) \cdot \operatorname{det} F_{a}(z)$, so that $\operatorname{det} F_{a}(z)$ $=b^{m(n-1)}(z)$. By virtue of Lemma 3.2, $F_{a}$ can be factored in the form $F_{a}=\prod_{j=m+1}^{m n} F_{j}$. Let $E_{k}=\prod_{j=1}^{k} F_{j}(k \leqq m n)$, and let $l_{k} \sim E_{k}$ (so that $l_{m}=l$, and $\left.l_{m n}=l_{b}\right)$. From Theorem B, we have $l_{k-1} \subseteq l_{k}$. Since no $F_{j}$ is constant, we have $l_{k-1} \subset l_{k}$. By use of induction, we see that $\operatorname{dim} l_{k}$ $\geqq k$, and also that $\operatorname{dim} l_{p+k} \geqq \operatorname{dim} l_{p}+k,(k+p \leqq m n)$. If $\operatorname{dim} l_{k}>k$ for some fixed $k$, then $\operatorname{dim} l_{m n} \geqq \operatorname{dim} l_{k}+m n-k>m n$. But it follows from Lemma 3.3 that $\operatorname{dim} l_{b}=m n$. Thus, by contradiction, $\operatorname{dim} l_{k}=k$, $k=1, \cdots, m n$.

Proof of Lemma 2. Let $l$ contain (exactly) $m$ linearly independent exponentials with exponent $\lambda$, and let $l_{\lambda}$ be the span of these exponentials. From Theorem B, we have $F=F_{\lambda} F_{a}$. Taking determinants of both sides, we have $\operatorname{det} F=\operatorname{det} F_{\lambda} \cdot \operatorname{det} F_{a}$. According to Lemma 3.4 , det $F_{\lambda}=c b^{m}(z)$, so that $\operatorname{det} F$ has a zero of order at least $m$ at $z=-\bar{\lambda}$.

Conversely, we assume that $\operatorname{det} F$ has a zero of order $m$ at $z=-\bar{\lambda}$. According to Lemma 3.2, $F$ can be factored in the form $F=\left(\prod_{j=1}^{m} F_{j}\right) F_{a}$ for prime $F_{j}$ 's. Let $l_{m} \sim \prod_{j=1}^{m} F_{j}$. Since det $\prod_{j=1}^{m} F_{j}=b^{m}(z)$, it follows from Lemma 3.4 that $l_{m}$ contains $m$ linearly independent exponentials with exponent $\lambda$. Then by Theorem $\mathrm{B}$, we have $l_{m} \subseteq l$, which completes the proof.

Proof of Lemma 3. Let $l$ contain an exponential $p(x) e^{i \lambda x}$ of order $m$. Since $\left(T_{s}-e^{i \lambda s}\right) p(x) e^{i \lambda x}(\in l)$ is an exponential of order $m-1$, we see that $l$ contains at least $m+1$ linearly independent exponentials with exponent $\lambda$. By virtue of Lemma $2, \beta(z)$ has a zero of order at least $m+1$, so that

$$
\left(\beta(-\bar{\lambda}) I \cdot h_{v}(-\bar{\lambda}), u\right)^{(k)}=0, \quad \text { for } k=0, \cdots, m .
$$

It then follows from equation (3.3) that all exponentials with exponent $\lambda$ of order $m$ or less are contained in $l_{\beta}$.

\section{BIBLIOGRAPHY}

1. A. Beurling, On two problems concerning linear transformations in Hilbert space, Acta Math. 81 (1949), 239-255.

2. P. R. Halmos, Shifts on Hilbert space, J. Reine Angew. Math. 208 (1961-1962), 102-112. 
3. H. Helson and D. Lowdenslager, Prediction theory and Fourier series in several variables, Acta Math. 99 (1958), 165-202.

4. K. Hoffman, Banach spaces of analytic functions, Prentice-Hall, Englewood Cliffs, N. J., 1962.

5. P. D. Lax, Translation invariant spaces, Acta Math. 101 (1959), 163-178.

6. - Translation invariant spaces, Proc. Internat. Sympos. on Linear Spaces (Jerusalem, 1960), Jerusalem Acad. Press, Jerusalem; Pergamon, Oxford, 1961, pp. 299-306.

7. V. B. Potapov, The multiplicative structure of J-contractive matrix functions, Amer. Math. Soc. Transl. (2) 15 (1960), 131-244.

Courant Institute of Mathematical Sciences, New York University 